\title{
Bioactive Sheath/Core Nanofibers Containing Olive Leaf Extract
}

\author{
GAMZE DOĞAN, ${ }^{1 *}$ GÜLDEMET BAŞAL, ${ }^{2}$ OĞUZ BAYRAKTAR,${ }^{3}$ FİGEN ÖZYILDIZ, ${ }^{4}$ ATAÇ UZEL, ${ }^{4}$ AND \\ IPEK ERDOGAN ${ }^{5}$ \\ ${ }^{1}$ Department of Materials Science and Nanotechnology Engineering, Uşak University, Uşak 64200, Turkey \\ ${ }^{2}$ Department of Textile Engineering, Ege University, Izmir 35100, Turkey \\ ${ }^{3}$ Department of Chemical Engineering, Ege University, İzmir 35100, Turkey \\ ${ }^{4}$ Department of Biology, Ege University, Izmir 35100, Turkey \\ ${ }^{5}$ Department of Bioengineering, Izmir Institute of Technology, İzmir 35430, Turkey
}

\begin{abstract}
KEYWORDS coaxial electrospinning; active agent release profile; antimicrobial activity; antioxidant activity
\end{abstract}

\begin{abstract}
This study aimed at producing silk fibroin (SF)/hyaluronic acid (HA) and olive leaf extract (OLE) nanofibers with sheath/core morphology by coaxial electrospinning method, determining their antimicrobial properties, and examining release profiles of OLE from these coaxial nanofibers. Optimum electrospinning process and solution parameters were determined to obtain uniform and bead-free coaxial nanofibers. Scanning electron microscopy and transmission electron microscopy (TEM) were used to characterize the morphology of the nanofibers. The antimicrobial activities of nanofibers were tested according to AATCC test method 100. Total phenolic content and total antioxidant activity were tested using in vitro batch release system. The quality and quantity of released components of OLE were determined by high-performance liquid chromatography. The changes in nanofibers were examined by Fourier-transform infrared spectroscopy. Uniform and bead-free nanofibers were produced successfully. TEM images confirmed the coaxial structure. OLE-loaded nanofibers demonstrated almost perfect antibacterial activities against both of gram-negative and gram-positive bacteria. Antifungal activity against C. albicans was rather poor. After a release period of 1 month, it was observed that $\sim 70-95 \%$ of the OLE was released from nanofibers and it was still bioactive. Overall results indicate that the resultant shell/core nanofibers have a great potential to be used as biomaterials. Microsc. Res.

Tech. 79:38-49, 2016. @ 2015 Wiley Periodicals, Inc.
\end{abstract}

\section{INTRODUCTION}

One of the widely used methods for producing nanofiber webs for biomedical applications is electrospinning. With the increasing interest in electrospinning by academic world, it has become a widely used technique to produce various nanofiber webs for a diverse set of applications. Ease of fiber manufacturing from lots of polymers has played a significant role in its popularity. Natural biopolymer nanofibers can also be fabricated rather easily by electrospinning. One of the natural biopolymers used in this study is silk fibroin (SF). Besides its electrospinnability, biocompatibility, good mechanical, and anti-inflammatory properties of SF make it indispensable for tissue scaffold and wound dressing applications (Wang et al., 2015; Altman et al., 2003). Although there are some studies in the literature including characterization (such as SEM, XRD, FTIR, and so on) (Li et al., 2006; Chen et al., 2006; Jin et al., 2002), and in vitro and in vivo evaluation of SF nanofibers (Min et al., 2004; Jin et al., 2004; Kim et al., 2005; Li et al., 2012), there are only limited number of articles in the literature about coaxial electrospinning of SF or silk sericin nanofibers (Wang et al., 2011; Hang et al., 2012). The other natural polymer used in this study is hyaluronic acid (HA). Nanosized HA is a promising material in tissue engineering. There are a few studies involving electrospinning of $\mathrm{HA}$ alone (Um et al., 2004; Young, 2006). Very high viscosity of HA makes its electrospinning difficult. To overcome this difficulty, HA was blended with other polymers (Ji et al., 2006; Fischer et al., 2012), or a blower and heater were integrated to the fiber formation zone (Um et al., 2004).

Coaxial electrospinning is a modified method that allows production of nanofibers with sheath/core morphology (Moghe, 2008). While a single nozzle (or needle) is used in traditional electrospinning method, coaxial electrospinning method utilizes two needles that coaxially placed to the feeding unit. This provides production of sheath/core structured nanofibers by feeding sheath solution and core solution throughout two different channels.

Previous studies suggest that electrospinning is as an effective way to produce nanofibers that can provide

\footnotetext{
*Correspondence to: G. Doğan; Department of Materials Science and Nanotechnology Engineering, Uşak University, 64200 Uşak, Turkey.

E-mail: gamze.dogan@usak.edu.tr; dogangamze@gmail.com

REVIEW EDITOR: Prof. Alberto Diaspro

Received 23 September 2015; accepted in revised form 31 October 2015

DOI 10.1002/jemt.22603

Published online 2 December 2015 in Wiley Online Library (wileyonlinelibrary.com).
} 
release of various compounds such as proteins, drugs, or active agents. One way to produce these compound loaded nanofibers is traditional electrospinning method. In traditional method, compound-loaded polymer nanofibers are produced by directly blending compound with polymer solution and then performing electrospinning (Shao et al., 2011; Jin et al., 2013; Loh et al., 2010; Chew et al., 2005; Xu et al., 2006; Okuda et al., 2010). Shao et al. (2011) stressed the potential uses of polymer composite nanofibers containing green-tea polyphenols in cancer treatments. Jin et al. (2013) studied electrospinning of a polymer solution including four different plant extracts. According to the results of study, plant extracts + polymer nanofibers were suitable for skin tissue engineering. The other way to produce compoundloaded nanofibers is coaxial electrospinning method. As pointed out by Jiang et al.'s review, encapsulation of compounds in core region of coaxial nanofiber is advantageous in terms of avoiding harmful effects of strong organic solvents used in preparing sheath solutions on compounds and obtaining structures enabling sustained release (Jiang et al., 2014). Fabrication of sheath/core nanofibers concluding drugs, genes, and proteins in core regions are reported by several researchers (Jiang et al., 2014; So and Mo, 2011; Saraf et al., 2010).

Antimicrobial and antioxidant natural compounds such as medicinal plants have been used for therapeutic purpose for thousands of years. In the last decades, researchers focused on these natural compounds and particularly development and potential uses of new products incorporated with these compounds. Antioxidant and antimicrobial activities of various plants were investigated by several researchers. Different parts of plants such as leaves, fruits, and oils obtained from the plants were taken into consideration (Briante et al., 2002; Visioli and Galli, 1998). Olive tree (Olea europaea) - that is, one of these antimicrobial and antioxidative plants-is commonly found in the vicinity of the Mediterranean and European. Olive leaf extract (OLE) has a very rich phenolic content. Phenolics are well-known antimicrobial agents. Basically, olive leaf includes 5 groups of contents: oleuropeosides, flavones, flavonols, flavan-3-ols, and phenolic acids (BenaventeGarcia et al., 2000). This rich phenolic content makes OLE antimicrobial and antioxidant (Luis et al., 2012; Altıok et al., 2008). OLE provides protection against viruses, bacteria, and fungi; and it has therapeutic effects on diseases (Markin et al., 2003; Sudjana et al., 2009).

The aim of this study was the production and characterization of OLE-loaded biopolymer-based coaxial nanofibers. For this purpose, initially uniform and bead-free coaxial nanofiber structure was obtained selecting optimum process and solution parameters. Subsequently, antimicrobial activities and the release profiles of OLE-loaded nanofibers were revealed. To the best of our knowledge, sheath/core nanofibers produced from biopolymers loaded with plant extracts have not yet been reported in the literature.

\section{MATERIALS AND METHODS Materials}

Raw Bombyx mori silk was obtained from Bursa Institute for Silkworm Research (Bursa, Turkey). Sodium carbonate, calcium chloride, ethanol, bromophe- nol blue, and acetonitrile were purchased from SigmaAldrich (Steinheim, Germany). HA sodium salt (from Streptococcus equi) and Saponin were supplied from Sigma (St.Louis, MO, USA). OLE was kindly provided from DUAG Natural Products (Izmir, Turkey) in powder form. Formic acid, Mueller-Hinton agar, Sabouraud dextrose agar, Tween 80, lecithin, folin-ciocalteu reagent, gallic acid, and acetic acid were obtained from Merck (Darmstadt, Germany). Phosphate-buffered saline (PBS) ( $\mathrm{pH}$ 7.2) was obtained from Gibco ${ }^{\mathrm{TM}}$ (Carlsbad, CA, USA). Trolox (6-hydroxy-2,5,7,8,-tetramethylchroman-2carboxylic acid), potassium persulfate $\left(\mathrm{K}_{2} \mathrm{O}_{8} \mathrm{H}_{8}\right)$, and ABTS (2,2'-azinobis(3-ethylbenzothiazoline-6-sulphonic acid)) were obtained from Fluka (Buchs, Switzerland).

\section{Preparation of Aqueous Silk Fibroin Solution}

Silk was treated with boiling aqueous solution of $0.05 \%$ sodium carbonate (50 times $\mathrm{v} / \mathrm{w}$ ) for $30 \mathrm{~min}$. This treatment was repeated three times. The degummed silk was rinsed in distilled water several times and left for drying at room temperature. Aqueous SF solution was obtained after dissolving $1.2 \mathrm{~g} \mathrm{SF}$ in $20(\mathrm{v} / \mathrm{w}) \mathrm{CaCl}_{2} /$ distilled water/ethanol (molar ratio 1:8:2) by stirring at $78^{\circ} \mathrm{C}$ for $2 \mathrm{~h}$ and dialyzed at $+4^{\circ} \mathrm{C}$ for 3 days to remove neutral salts. Dialyzed solution were put in Petri dishes and dried under vacuum to obtain SF films which was used in the preparation of electrospinning solution.

\section{Preparation of Electrospinning Solution}

Polymer solutions were prepared by stirring overnight. SF (shell) was dissolved in formic acid and HA and OLE (core) dissolved in distilled water. Optimum solution properties were determined as $15 \%$ (w/v) SF/ formic acid for shell solution and $0.5 \%(\mathrm{w} / \mathrm{v}) \mathrm{HA} / \mathrm{dis}-$ tilled water during preliminary studies. The maximum OLE concentrations that allowed uniform coaxial nanofiber formation were 12 and $15 \%(\mathrm{w} / \mathrm{v})$. Coaxial nanofibers without OLE $(0 \%(\mathrm{w} / \mathrm{v}))$ were prepared as control groups for tests.

\section{Electrospinning}

Two syringe pumps (New Era Pump Systems NE300, USA) and a high-voltage power supply (Iseg Spezialelektronik GmbH, Rossendorf, Germany) were used for electrospinning coaxial nanofibers. Coaxial electrospinning setup and coaxial needle used in this study are given in Figure 1. High-magnification photos of the needle tip were also taken for the observation of stable coaxial electrospinning jet.

The coaxial needle was composed of an outer needle of $14 \mathrm{G}$ (id $0.9 \mathrm{~mm}$ - od $1.4 \mathrm{~mm}$ ) and an inner needle of $24 \mathrm{G}$ (id $0.5 \mathrm{~mm}$ - od $0.7 \mathrm{~mm}$ ). Rotating drum was used as collector to improve solvent evaporation and uniform coaxial fiber production. The surface velocity of the rotating drum was $188.5 \mathrm{~m} / \mathrm{min}(3.1 \mathrm{~m} / \mathrm{s})$. For the ease of fiber collection from the surface of the rotating drum, the drum was coated with a polyester nonwoven fabric. The distance was fixed at $10 \mathrm{~cm}$. Flow rates of sheath and core polymer solutions were determined as 8 and $2 \mu \mathrm{L} / \mathrm{min}$, respectively. Applied voltages for all nanofiber samples were in the range of $15-20 \mathrm{kV}$. After $20 \mathrm{~h}$ of spinning, nanofiber webs were removed from the collector. Coaxial nanofibers webs that contain 


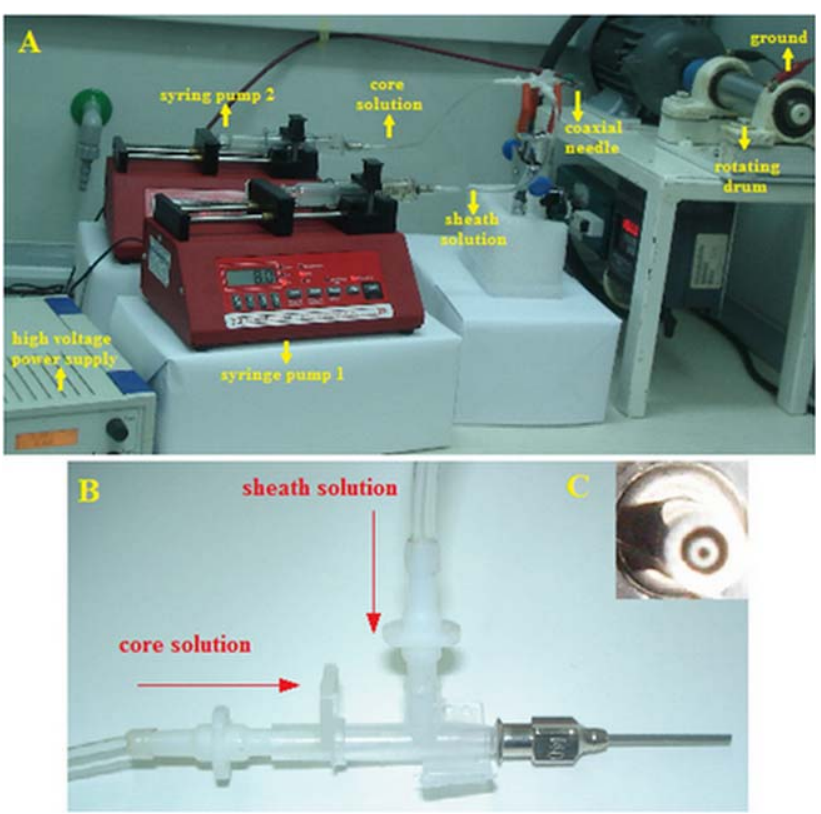

Fig. 1. (a) Coaxial electrospinning setup. (b) Coaxial needle designed. (c) The cross-section of the coaxial needle. [Color figure can be viewed in the online issue, which is available at wileyonlinelibrary.com.]

$15 \%, 12 \%$, and $0 \%$ of OLE in their core solution were named as OLE-15, OLE-12, and OLE-0 for ease of expression.

\section{Characterization of Nanofibers}

Scanning electron microscope (SEM) images were taken (Philips XL 30S FEG and FEI Quanta FEG, Eindhoven, Holland) for morphological analysis. Before SEM analysis, the samples were coated by gold sputtering in an argon atmosphere. The average fiber diameter (AFD) and diameter distribution were determined by choosing 100 fibers randomly from SEM images and using ImageJ measurement and visualization software. TEM images of the electrospun coaxial nanofibers were taken at a voltage of $80 \mathrm{kV}$ (FEI Tecnai Biotuin $\mathrm{G}^{2}$ Spirit, Oregon, USA). To obtain contrast between shell and core morphology, bromophenol blue was added to core solution $(0.3 \%(\mathrm{w} / \mathrm{v}) \mathrm{HA}$ in water $)$ with a ratio of $5 \%(\mathrm{w} / \mathrm{v})$. Electrospinning was performed on a Holey carbon film coated 200 mesh copper TEM grids (Electron Microscopy Sciences, Hatfield, PA, USA).

\section{Antimicrobial Activity Analysis}

The antimicrobial activities of coaxial nanofiber webs including different concentrations of OLE were evaluated according to AATCC test method 100. Coaxial nanofiber webs without OLE were used as negative control. Webs were tested against Methicillin-resistant Staphylococcus aureus ATCC 43300, Escherichia coli 0157H7 RSKK232, and Candida albicans DSMZ5817. Test bacteria were grown in Mueller-Hinton agar and C. albicans were grown in Sabouraud dextrose agar at $37^{\circ} \mathrm{C}$ for $24 \mathrm{~h}$. Activated microorganisms were washed with $0.85 \%$ sterile physiological saline solution (SPSS) two times and initial inoculations were prepared by diluting the microorganisms in SPSS to obtain optical densities corresponding to 0.5 McFarland at $600 \mathrm{~nm}$ using a spectrophotometer (Varian Cary 50). Five square centimeters of nanofiber webs were prepared in aseptic conditions and taken in sterile falcon tubes. After saturating each nanofiber web with $1 \mathrm{~mL}$ of suspension of test microorganisms, one series of samples were diluted as soon as possible after inoculation and plated at "0" contact time. Another series of the samples were incubated at $37^{\circ} \mathrm{C}$ for $1 \mathrm{~h}$ and then plated on appropriate media. Antimicrobial activity test was stopped after the desired contact time period using $1 \mathrm{~mL}$ neutralizing solution composed of $30 \mathrm{~g} / \mathrm{L}$ Tween $80+30 \mathrm{~g} /$ $\mathrm{L}$ Saponin, $3 \mathrm{~g} / \mathrm{L}$ lecithin in both series. All samples were plated in appropriate culture media in duplicate. When the incubation was completed at $37^{\circ} \mathrm{C}$ for $48 \mathrm{~h}$, colony counts were detected. The quantitative results were expressed in terms of colony forming units per milliliter $(\mathrm{CFU} / \mathrm{mL})$. The reduction rate in the number of test microorganisms was calculated by using the following formula; $R(\%)=(B-A) / B \times 100$, where $R$ is the percent reduction rate, $A$ is the number of microorganisms recovered from the inoculated nanofibers over $1 \mathrm{~h}$, and $B$ is the number of microorganisms recovered from the inoculated nanofibers at zero contact time. All experiments were done in duplicates and mean values were used in evaluation.

\section{Release Test Procedure}

The release of OLE from the coaxial nanofiber web was performed by determining the diffusion of the phenolic content and antioxidant active compound in a release medium, which was prepared by diluting phosphate-buffered saline (PBS) ( $\mathrm{pH} 7.2) 10$ times. Nanofiber web samples of $10 \mathrm{mg}(\sim 2 \times 2 \mathrm{~cm})$ were put into 12 well plates and $5 \mathrm{~mL} 1 \times$ PBS was added into each well. The test duration time was 1 month. Samples were taken from the release medium at determined time intervals $(0,2,4,6,24,48$, and $72 \mathrm{~h} ; 1,2$, 3 , and 4 weeks) and the amount of OLE release from the nanofiber web to medium was quantified by using total phenolic content and total antioxidant activity analyses. At the end of 1 month, release mediums were used for HPLC analysis to determine the quantities and qualities of OLE components released from coaxial nanofibers.

\section{Total Phenol Content Analysis}

Total phenol content of OLE released from nanofibers was determined by Folin-Ciocalteu assay. Hundred microliters of Folin-Ciocalteu reagent (diluted 1:10 with water) was mixed with $20 \mu \mathrm{L}$ liquid sample. To stop the reaction between sample and agent, $80 \mu \mathrm{L}$ $\mathrm{Na}_{2} \mathrm{CO}_{3}(7 \%$ (w/v)) was added. Samples were then kept under dark condition for $1 \mathrm{~h}$ and analyzed by UV spectrophotometer (Thermo Scientific Multiskan Spectrum, Waltham, MA, USA) at $725 \mathrm{~nm}$. Gallic acid was used in calibration and total phenol contents were given in terms of Gallic Acid Equivalent (GAE). Total phenolic content of OLE used in this study was measured by dissolving OLE in PBS using triplicate sets of three dilution ratios. 
Extraction of phenolic content from nanofibers was performed by a mini bead beater (Biospec Products, Bartlesville, OK, USA) to determine the total phenolic content of coaxial nanofibers containing OLE. Twenty micrograms of sample, $1 \mathrm{~mL}$ PBS, and $0.1 \mathrm{~g}$ ZirconiaSilica beads $(0.1 \mathrm{~mm}$ in diameter, Biospec Products, Bartlesville, OK, USA) were added in microvial of $2 \mathrm{~mL}$ capacity. The rate of oscillation was adjusted to 2500 oscillations/min. Samples were taken at every 3 min and the process was carried on till no phenolic content has been detected.

\section{Total Antioxidant Activity Analysis}

Total antioxidant activity of nanofibers was assessed by using the Trolox Equivalent Antioxidant Capacity (TEAC) assay. The radical scavenging ability of OLE released from nanofibers was determined in terms of Trolox equivalent antioxidant capacity. ABTS was diluted dissolving in water (to $7 \mathrm{mM}$ concentration) and activated with $2.45 \mathrm{mM}$ potassium persulphate to generate $\mathrm{ABTS}^{+}$radical. The $\mathrm{ABTS}^{+}$solution was kept in the dark under room temperature for $16 \mathrm{~h}$ for the reaction to be completed. Measurement was made by microplate reader (Thermo Varioskan Flash, Waltham, MA, USA). The $\mathrm{ABTS}^{+}$solution was diluted with ethanol to an absorbance of $0.70( \pm 0.03)$ at $734 \mathrm{~nm}$. Ten microliters of sample was added to $\mathrm{ABTS}^{+}$ solution of $2 \mu \mathrm{L}$ and percent inhibition values were recorded according to the decrease in absorbance. In order to determine the total antioxidant capacity of OLE-loaded coaxial nanofibers, the extracts from nanofiber samples prepared as in case of total phenol content analysis were used. The total antioxidant capacity (TAOC) of OLE used in this study was determined by triplicate sets of three dilution ratios as well.

\section{High-Performance Liquid Chromatography (HPLC) Analysis}

High-performance liquid chromatography (HPLC, Hewlett-Packard Series HP 1100 equipped with a diode array detector) was used for determination of OLE components released from coaxial nanofibers. Samples taken from released medium at the end of 1 month were fed to system after filtering through 0.45 $\mu \mathrm{m}$ syringe filter. The stationary phase was a C18 LiChrospher 100 analytical column $(250 \times 4 \mathrm{~mm}$ i.d. $)$ with a particle size of $5 \mu \mathrm{m}$ thermostated at $30^{\circ} \mathrm{C}$. The flow rate was $1 \mathrm{~mL} / \mathrm{min}$, and the absorbance changes were monitored at $280 \mathrm{~nm}$. The mobile phases for chromatographic analysis were (A) acetic acid/water (2.5:97.5) and (B) acetonitrile. A linear gradient was run from $95 \% \mathrm{~A}$ and $5 \% \mathrm{~B}$ to $75 \% \mathrm{~A}$ and $25 \% \mathrm{~B}$ during $20 \mathrm{~min}$; it changed to $50 \% \mathrm{~A}$ and $\mathrm{B}$ in $20 \mathrm{~min}$ (40 min, total time); in 10 min it changed to $20 \% \mathrm{~A}$ and $80 \% \mathrm{~B}$ (50 min, total time), after re-equilibration in $10 \mathrm{~min}$ (60 min, total time) to initial composition. Phenolic components in released medium were determined by comparing retention times of each component with those of known standards.

\section{Fourier-Transform Infrared Spectroscopy (FTIR) Analysis}

FTIR spectroscopy analysis was made to observe the chemical changes within the coaxial nanofibers before and after release tests. IR spectra were measured using $\mathrm{KBr}$ pellets on a Shimadzu (Japan) 8400S FT-IR over the range of $400-4000 \mathrm{~cm}^{-1}$ with a resolution of $2 \mathrm{~cm}^{-1}$. To form pellets $\sim 2.0 \mathrm{mg}$ sample was mixed with $120 \mathrm{mg} \mathrm{KBr}$ and the mixture was pressed in a $13 \mathrm{~mm}$ pellet die under a pressure of $6,000 \mathrm{psi}$.

\section{Determination of Weight Loss}

To determine the weight loss during the release test, $10 \mathrm{mg}$ of the nanofiber webs were taken and the weights of these samples were re-measured after the release test. In order to remove all the PBS in the nanofibers, samples were washed with distilled water two times; then dried in incubator at $30^{\circ} \mathrm{C}$ for $4 \mathrm{~h}$ and their weights were measured. Tests were performed in triplicate.

$$
\text { Weight loss }(\%)=\frac{w_{1}-w_{2}}{w_{1}} * 100
$$

Where $\mathrm{w}_{1}$ is the weight of nanofiber webs before release test and $\mathrm{w}_{2}$ is the weight of nanofiber webs after release test.

\section{RESULTS \\ Results of Electrospinning}

During electrospinning process, the formation of stable coaxial electrospinning jet can be seen in the highmagnification photos taken under three different voltages $(0,20$, and $30 \mathrm{kV})$ (Figure 2).

The SEM images given in Figure 3 show uniform and bead-free nanofibers produced with determined parameters. The diameters of nanofibers followed the normal distribution. The nanofibers without OLE had the lowest average fiber diameter. As the OLE concentration increased the average fiber diameter increased as well.

TEM images of nanofibers were taken to observe the coaxial fiber formation. Sufficient contrast between two polymers was accomplished by Bromophenol Blue. As seen in Figure 4, properly arranged core region demonstrates a uniform coaxial nanofiber. The core diameter was $110.73 \mathrm{~nm}$ and thickness of the sheath was $156.96 \mathrm{~nm}$.

\section{Antimicrobial Activities of Nanofibers}

Since OLE is a well-known antimicrobial compound, antimicrobial activities of nanofiber webs were tested to determine whether antimicrobial activities were retained after electrospinning. Antimicrobial activities against tested microorganisms are given in Figure 5. OLE containing coaxial nanofiber webs exhibited a perfect antimicrobial property as seen from the graphs. The reduction rate in the number of $S$. aureus and $E$. coli were between the range of $99.98-100.00 \%$ for OLE-12 and OLE-15. However, the antifungal effects of nanofibers were rather poor. Approximately $27 \%$ of C. albicans was eliminated even with the maximum OLE content of nanofibers. This value was only $16 \%$ for nanofiber webs named as OLE-12.

\section{Total Phenolic Contents of Nanofibers}

The OLE used in this study contains a total phenolic content of $14.04 \pm 0.83 \mathrm{mg} \mathrm{GAE} / \mathrm{g}$ OLE. Figure 6 shows the total phenolic contents of OLE-15 and OLE-12 

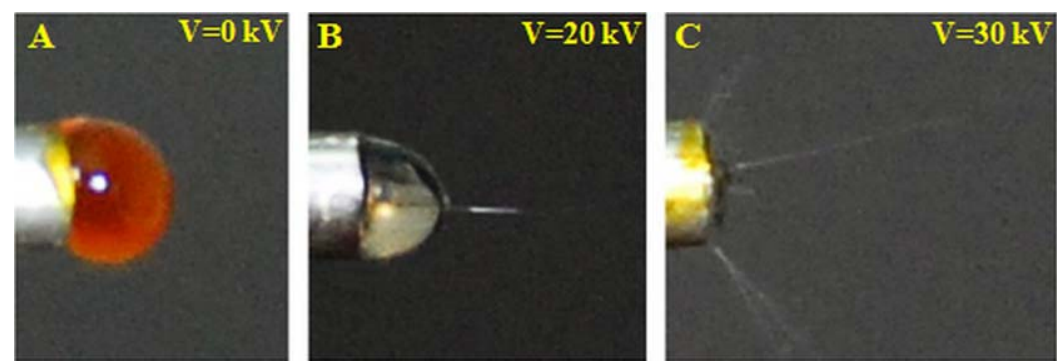

Fig. 2. Images of SF (sheath)/HA + bromophenol blue (core): (A) droplet at the tip of the needle, (B) stable compound cone and jet, and (C) disappeared cone and splitted jet by the effect of high voltage. [Color figure can be viewed in the online issue, which is available at wileyonlinelibrary.com.]

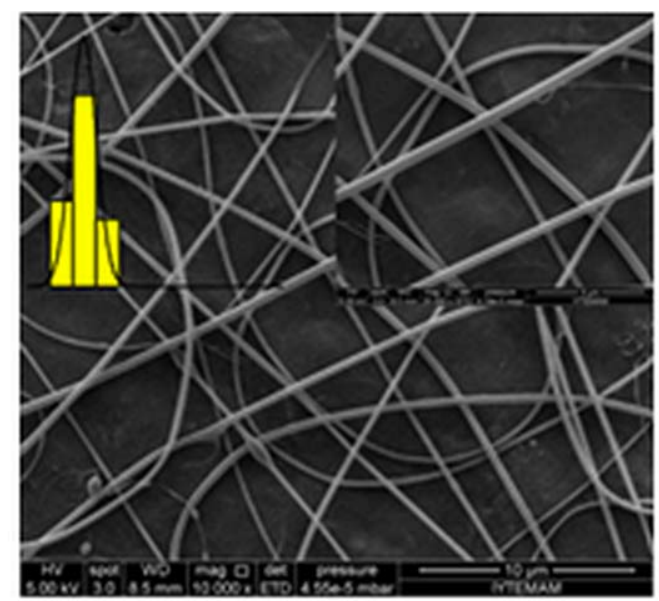

(a) $279.07 \pm 62.54$

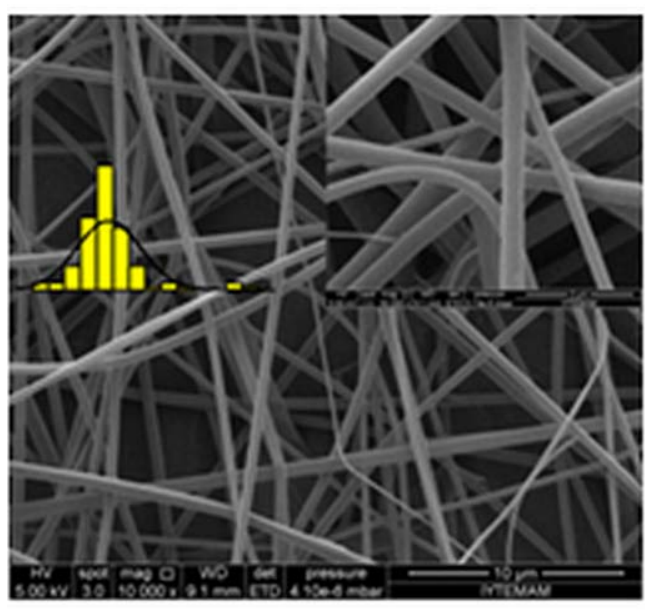

(b) $468.19 \pm 161.51$

Fig. 3. SEM images of coaxial nanofibers obtained at constant sheath flow rate $(8 \mu \mathrm{L} / \mathrm{min})$, core flow rate $(2 \mu \mathrm{L} / \mathrm{min})$, needle to collector distance $(10 \mathrm{~cm})$, and surface velocity of rotating drum $(3.14 \mathrm{~m} / \mathrm{s})$. Applied voltages for (a) OLE0: $15 \mathrm{kV}$, (b) OLE12: $20 \mathrm{kV}$, and (c)

nanofiber webs in terms of GAE. Columns show the instantaneous total phenolic contents of nanofiber webs arose due to the extraction using bead beater at

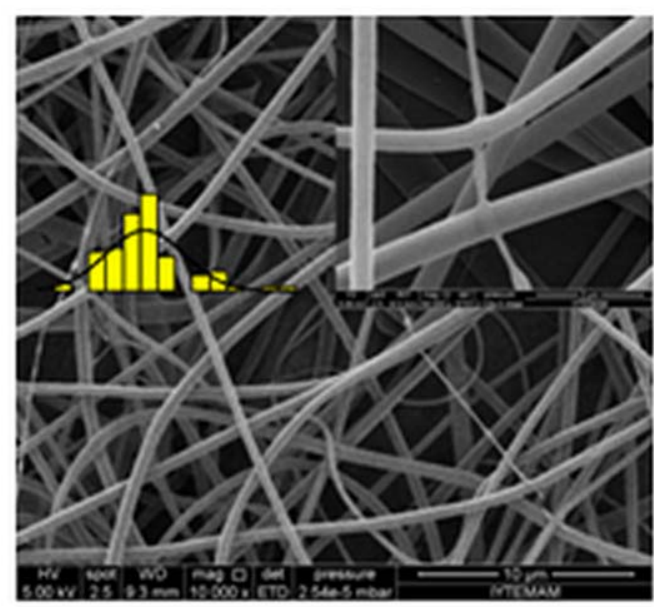

(c) $543.24 \pm 196.09$

OLE15: $19.5 \mathrm{kV})$ (magnifications: $10,000 \times$ and 50,000 $\times$ (right top)). [Color figure can be viewed in the online issue, which is available at wileyonlinelibrary.com.]

the end of determined periods. Cumulative values show the total phenolic contents arose at the end of extraction with bead beater test. It was observed that 


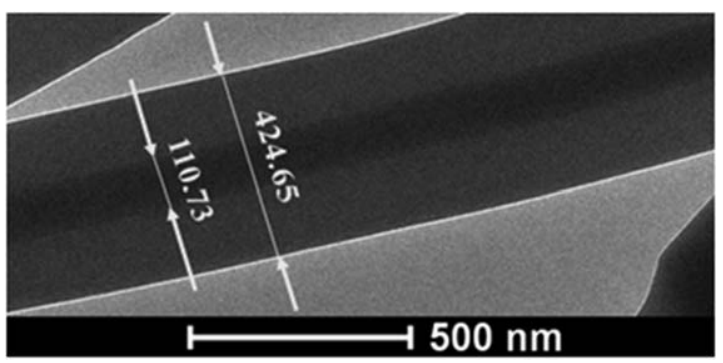

Fig. 4. TEM image of $15 \%(\mathrm{w} / \mathrm{v}) \mathrm{SF}($ sheath)/ $0.3 \%(\mathrm{w} / \mathrm{v}) \mathrm{HA}+5 \%$ $(\mathrm{w} / \mathrm{v})$ bromophenol blue (core) coaxial nanofibers.

A

S. aureus

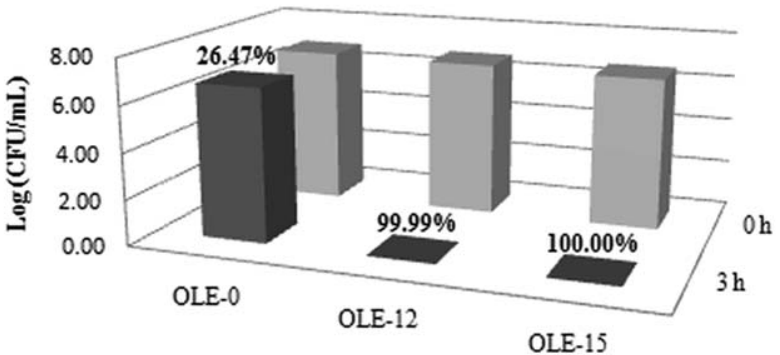

B

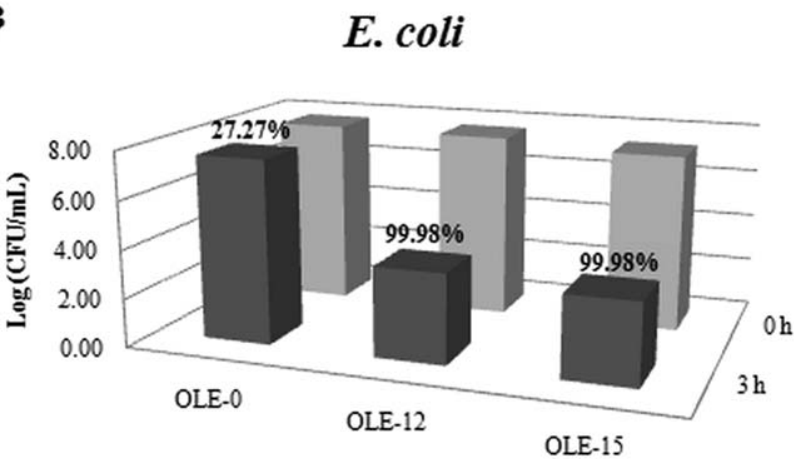

C

\section{C. albicans}

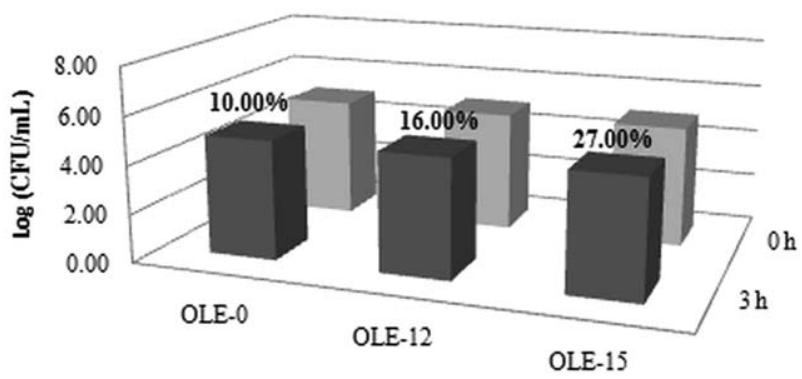

Fig. 5. Antimicrobial activities of coaxial nanofiber webs against (A) S. aureus, (B) E. coli, and (C) C. albicans.

OLE-15 nanofiber web had a total phenol content of $0.129 \mathrm{mg}$ GAE/g nanofiber, whereas OLE-12 nanofiber web had $0.101 \mathrm{mg}$ GAE/g nanofiber.

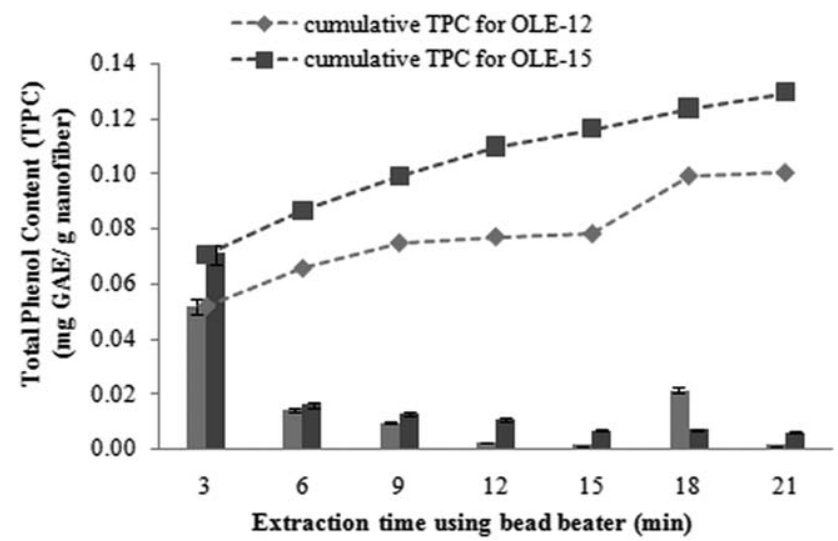

Fig. 6. GAE total phenolic contents of OLE-15 and OLE-12 nanofiber webs (columns show the instantaneous values for related extraction time using bead beater).

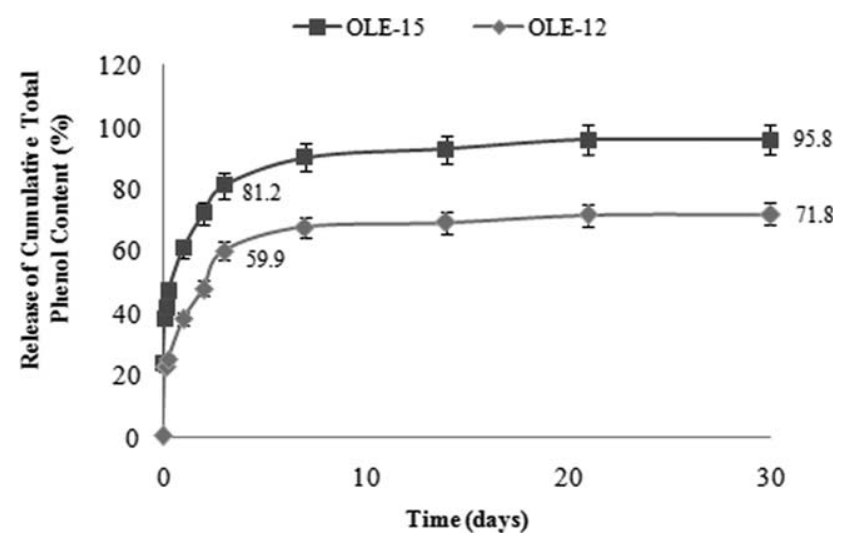

Fig. 7. Total phenol content batch release profiles of OLE-12 and OLE-15 nanofiber webs.

Figure 7 shows the total phenolic content release profiles of OLE-15 and OLE-12 nanofiber webs. It is seen from the figure that the sample OLE-15 lost $\sim 80 \%$ of its initial OLE content during the first $72 \mathrm{~h}$. Following initial release, a gradual release was observed. When the test was concluded by the end of 1 month, $95 \%$ of the total OLE content released which corresponds to $0.124 \mathrm{mg}$ GAE/g nanofiber. Sample OLE-12, on the other hand, lost $\sim 60 \%$ of its initial OLE during the first $72 \mathrm{~h}$, and after 1 month period, the remaining part had $\sim 30 \%$ of the total OLE content. Thus, the amount of released phenolic content for sample OLE-12 was $0.072 \mathrm{mg}$ GAE/g nanofiber.

\section{Total Antioxidant Capacity of Nanofibers}

Total antioxidant capacity of OLE used in this study was $11.62 \pm 1.22 \mathrm{mM}$ TEAC/g OLE. Total antioxidant capacities of bead-beated coaxial nanofibers were determined as in Figure 8. The results were given as difference between the control (OLE-0) and the samples (OLE-12 and OLE-15). The cumulative values show that OLE-15 and OLE-12 nanofiber webs had an antioxidant capacity of 2.74 and $2.15 \mathrm{mM} \mathrm{TEAC/g}$ nanofiber, respectively. 


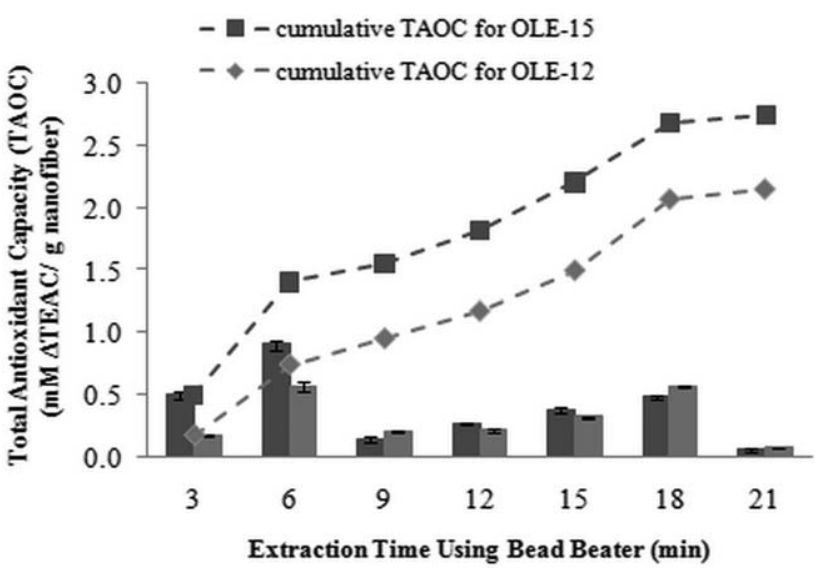

Fig. 8. Total antioxidant capacities of OLE-15 and OLE-12 nanofiber webs (columns show the instantaneous values for related extraction time using bead beater).

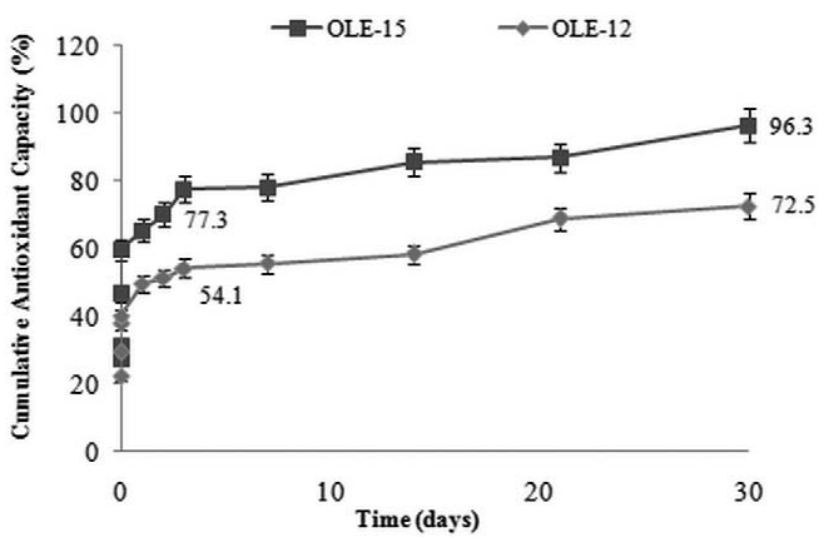

Fig. 9. Total antioxidant compound release profiles of OLE-15 and OLE-12 nanofiber webs.

Figure 9 shows the total antioxidant capacity of compounds released from nanofibers during 1 month. The released total antioxidant capacity from OLE-15 nanofiber webs was $2.64 \mathrm{mM}$ TEAC/g nanofiber. This means that $96.3 \%$ of OLE was released at the end of 1 month form OLE-15. The released total antioxidant capacity from OLE-12 nanofiber webs was $1.56 \mathrm{mM} \mathrm{TEAC} / \mathrm{g}$ nanofiber. This means $75.5 \%$ of OLE was released at the end of 1 month form OLE-12.

When the antioxidant capacity release profiles were examined (Fig. 9), it was observed that the OLE-15 lost $77.3 \%$ of its OLE content at the first $72 \mathrm{~h}$ and it released approx. $22 \%$ of the remaining OLE content during the period of $72 \mathrm{~h}$ to 1 month. OLE-12, on the other hand, lost $54 \%$ of its OLE content at the first $72 \mathrm{~h}$, and it released approx. $18 \%$ of the remaining OLE content during the period of $72 \mathrm{~h}$ to 1 month.

\section{Results of HPLC Analysis}

OLE used in this study was dissolved in PBS and analyzed by HPLC for the purpose of determining the components of extract. Each peak in the HPLC chromatogram (Fig. 10) that matches components of OLE is given in Table 1. Identification of peaks correspond-

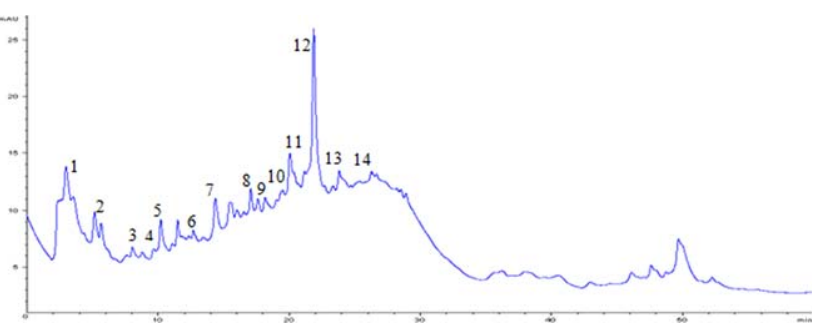

Fig. 10. HPLC chromatogram of OLE (1 mg/mL). [Color figure can be viewed in the online issue, which is available at wileyonlinelibrary.com.]

TABLE 1. Phenolic compounds released from OLE-15 and OLE-12 nanofibers

\begin{tabular}{lcccc}
\hline & & \multicolumn{3}{c}{ Peak area (\%) } \\
\cline { 3 - 5 } Component of & Peak & & & $\begin{array}{c}\text { OLE } \\
\text { dissolved } \\
\text { in PBS }\end{array}$ \\
\hline olive leaf & number & OLE-15 & OLE-12 & \\
Hydroxytyrosol & 1 & $\mathbf{1 0 . 6 9}$ & $\mathbf{1 0 . 2 9}$ & $\mathbf{2 . 0 8}$ \\
Tyrosol & 2 & $\mathbf{8 . 3 2}$ & $\mathbf{9 . 9 8}$ & $\mathbf{1 . 0 8}$ \\
Catechin & 3 & $\mathbf{4 . 2 2}$ & $\mathbf{4 . 0 7}$ & $\mathbf{0 . 3 7}$ \\
Caffeic acid & 4 & 0.73 & 0.99 & 1.18 \\
Vanillic acid & 5 & - & - & 0.71 \\
Vanillin & 6 & 0.35 & - & 2.45 \\
Rutin & 7 & 1.21 & 0.50 & 2.53 \\
Luteolin-7-glucoside & 8 & - & - & 2.06 \\
Verbascoside & 9 & 2.68 & - & 2.79 \\
Apigenin-7-glucoside & 10 & - & - & 2.04 \\
Diosmetin-7-glucoside & 11 & - & - & 5.78 \\
Oleuropein & 12 & 2.19 & 1.14 & 8.38 \\
Luteolin & 13 & 1.31 & 0.40 & 4.54 \\
Diosmetin & 14 & 0.81 & - & 1.55 \\
\hline
\end{tabular}

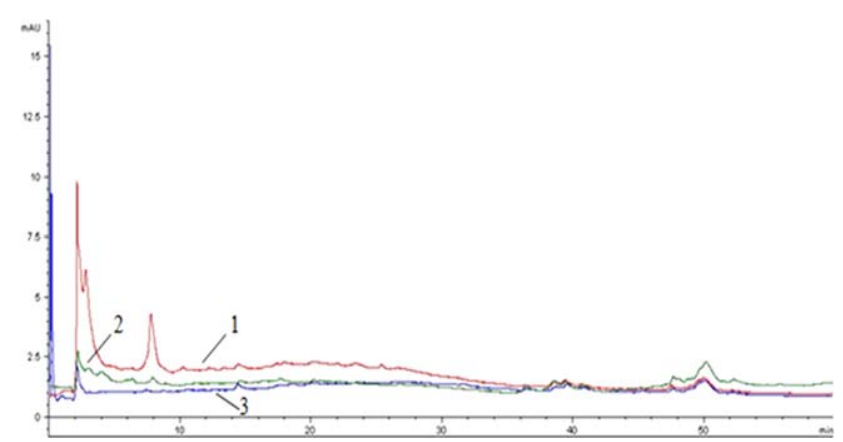

Fig. 11. HPLC chromatograms of the released medium from (1) OLE-15, (2) OLE-12, and (3) OLE-0 nanofiber webs at the end of 1 month. [Color figure can be viewed in the online issue, which is available at wileyonlinelibrary.com.]

ing to compounds in OLE was performed considering the retention times of standard compound. Percentages of phenolic contents were determined by the area under each peak.

After this procedure, analysis of compounds released from nanofibers was carried out. Figure 11 shows the HPLC chromatograms of the released medium from OLE-15, OLE-12, and OLE-0 nanofiber webs at the end of 1 month.

\section{Results of FTIR Analysis}

FTIR analysis was performed on samples before and after release test in order to examine the chemical 


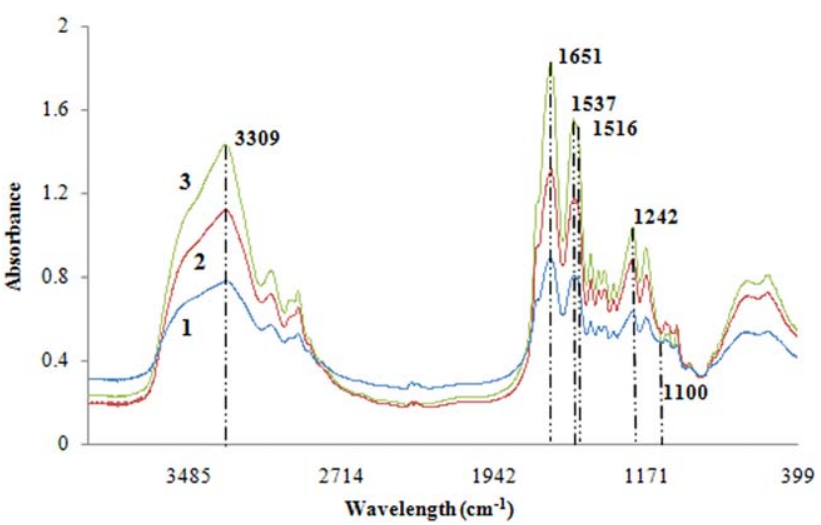

Fig. 12. FTIR spectra of (1) OLE-15, (2) OLE-12, and (3) OLE-0 coaxial nanofibers before release test. [Color figure can be viewed in the online issue, which is available at wileyonlinelibrary.com.]
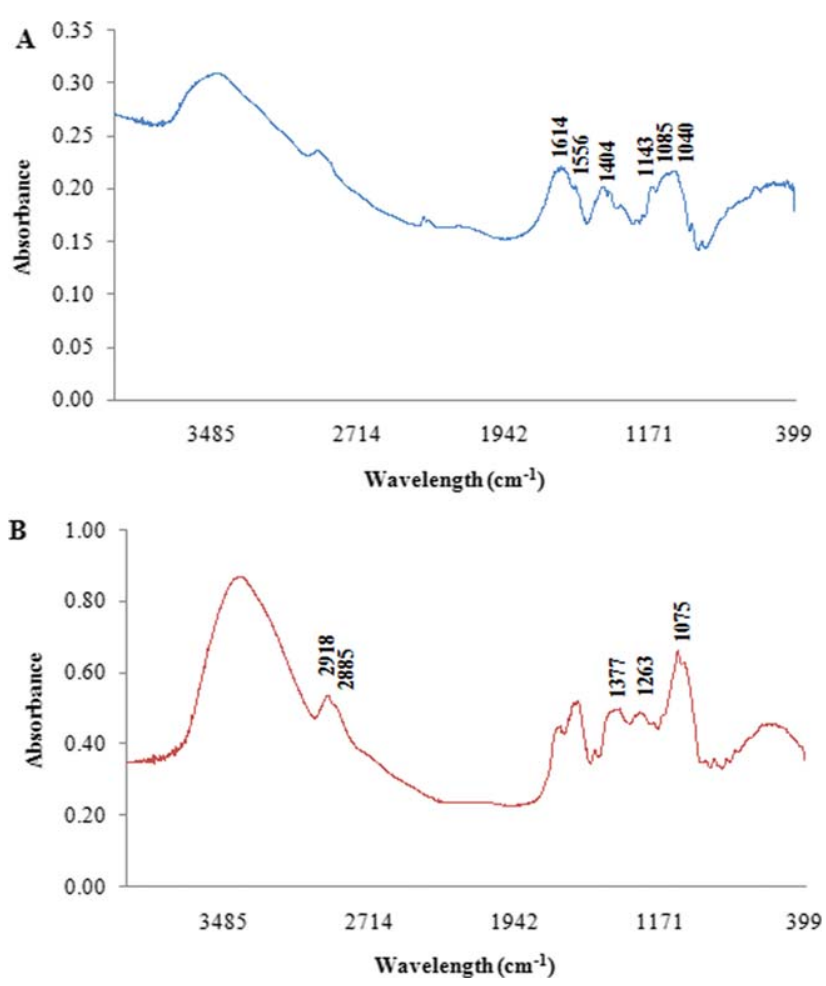

Fig. 13. FTIR spectrum of (a) hyaluronic acid sodium salt and (b) OLE used. [Color figure can be viewed in the online issue, which is available at wileyonlinelibrary.com.]

changes in coaxial nanofibers. Figure 12 shows the FTIR spectra of all nanofiber webs prepared. Since the most abundant component in the structure of nanofibers is SF, the FTIR spectrum of nanofibers seems like spectrum of SF.

As seen in the FTIR spectrum of HA sodium salt (Fig. 13a), the effect of HA on the absorbance bands of nanofibers is low. Bands at wavelengths of 3300, 3069, $1614,1556,1404,1143$, and $1040 \mathrm{~cm}^{-1}$ are originated from $\mathrm{O}-\mathrm{H}$ stretching, $\mathrm{NH}$ stretching, $\mathrm{C}=\mathrm{O}$ stretching, $\mathrm{NH}$ bending, $\mathrm{C}-\mathrm{O}$ stretching, $\mathrm{C}-\mathrm{O}-\mathrm{C}$ glycoside stretch-

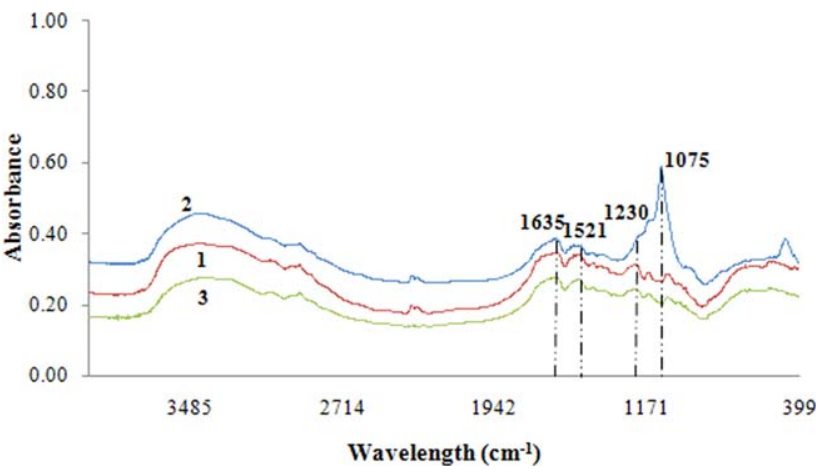

Fig. 14. FTIR spectra of (1) OLE-15, (2) OLE-12, and (3) OLE-0 coaxial nanofibers at the end of release test. [Color figure can be viewed in the online issue, which is available at wileyonlinelibrary. com.

ing, and $\mathrm{C}-\mathrm{OH}$ stretching of alcohols, respectively, as stated by Haxaire et al. (2003).

The characteristic bands of OLE can be seen in Figure 13b. Bands at wavelengths of 2918 and $2885 \mathrm{~cm}^{-1}$ are originated from $\mathrm{CH}$ stretching and bands at wavelengths of $1300-1100 \mathrm{~cm}^{-1}$ are originated from C-O stretching of phenols. The weak bands seen in spectra of OLE-15 and OLE-12 nanofibers were not observed in OLE-0 nanofibers (Fig. 14).

\section{Weight Loss of Nanofibers}

Weight loss was observed in all samples. This could be attributed to the release of phenolic components and hydrolytic degradation of polymers during 1 month. The weight losses were determined as 29.0, 36.7 , and $39.0 \%$ for OLE-0, OLE-12, and OLE-15 nanofibers, respectively.

\section{Morphological Changes in Nanofibers After Release Test}

As seen from the SEM images of nanofibers taken after release test (Fig. 15), swelling or fused structure is observed at nanofibers. The increases in AFDs were calculated by measuring diameters of randomly selected 40 fibers. The increases in nanofiber diameters were as follows: $357 \%, 143 \%$, and $161 \%$ for samples OLE-0, OLE-12, and OLE-15, respectively. The sample OLE-12 exhibited the minimum swelling.

\section{DISCUSSIONS}

The aim of this study was to prepare and characterize SF/HA sheath/core nanofiber webs including OLE and investigate the release of OLE from these nanofibers. The first step was coaxial electrospinning. In coaxial electrospinning, when the high voltage is applied to polymer solutions, sheath polymer solution is charged at first and then forms pending droplet. This droplet takes a form of cone due to the stress created by charge-charge repulsions. Then a jet is emerged. The stress is transferred from sheath solution to core solution. Core solution contributes to the formation of compound jet. The stable compound jet is defined as a jet that core solution is uniformly distributed in sheath solution. Solvent evaporation takes place during flight toward the collector, and solid 


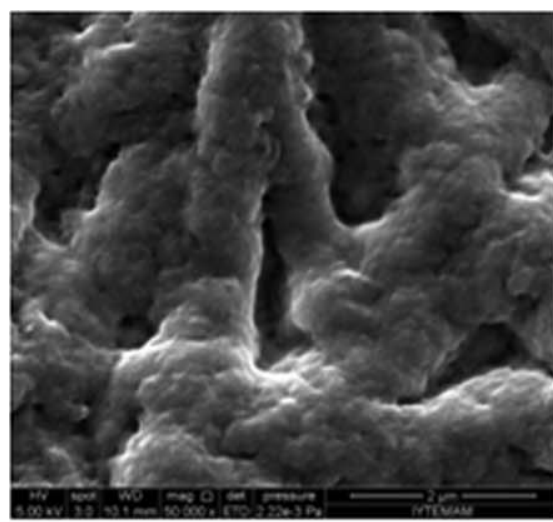

(a)

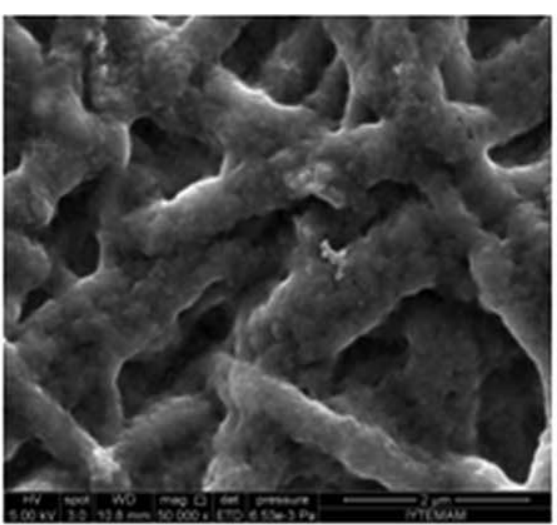

(b)

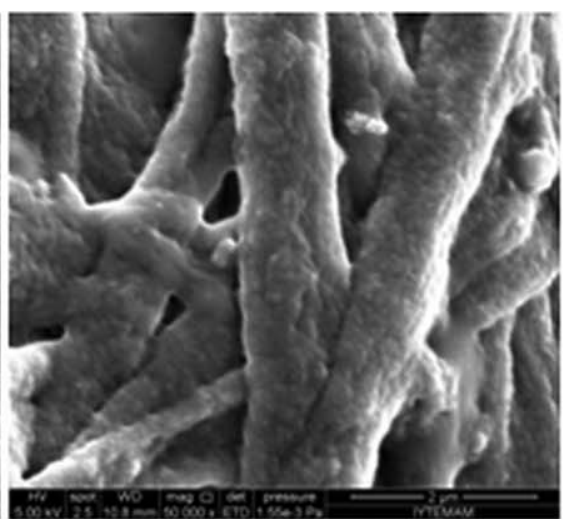

(c)

Fig. 15. SEM images of nanofibers after release test (a) OLE-0, (b) OLE-12, and (c) OLE-15 (magnification: $50,000 \times)$.

sheath/core structured nanofibers are formed (Moghe, 2008). There are specific critical voltage values for every polymer/solvent system. When this critical voltage is exceeded, the compound jet is splitted. As seen from the high-magnification photos of the tip of the needle (Fig. 2), a stable coaxial jet was observed at a voltage of $20 \mathrm{kV}$ (Fig. 2b). The critical voltage for this polymer solvent system was exceeded at a voltage of $30 \mathrm{kV}$ and cone was disappeared, multijets were created (Fig. 2c). When there was no applied voltage (Fig. $2 \mathrm{a})$, only the droplet was seen at the tip of coaxial needle. Since bromophenol blue acts as an indicator, a long contact time with formic acid turned its color to orange/yellow.

When the AFDs given in Figure 3 are considered, it is observed that OLE-0 nanofibers had the minimum and OLE-15 nanofibers had the maximum AFDs. Phenolic compounds added to polymer solutions increase electrical conductivity, and an increase in electrical conductivity results in lower AFD. Addition of phenolics also increases the solution viscosity since it causes an increase in solid volume percent. Optimum voltages for all nanofibers were determined considering the stable compound jet. Voltages of 15, 20, and 19.5 voltages were applied for OLE-0, OLE-12, and OLE-15 nanofibers, respectively. Since all other electrospinning parameters except OLE concentration and voltage are constant, it can be concluded that combined effect of OLE concentration and voltage resulted in an increase in AFDs of nanofibers.

Since the antimicrobial activities of nanofiber webs that have potential to be used as biomaterials are significant features, antimicrobial activities of OLEloaded sheath/core nanofiber webs were tested. Higher concentration of active agent means good antimicrobial activity. So, it was expected for the OLE-15 samples to show the highest growth inhibition ability against both bacteria and fungus. Significant antibacterial activities were observed for both OLE-12 and OLE-15 nanofiber webs. It is stated in the literature that higher concentration values of plant extract is required for the inhibition of growth of fungi compared to bacteria since the characteristics of cells are differ- ent from each other (Markin et al., 2003). So, if OLEloading capacity of the nanofibers could be increased, there would be a possibility to prepare nanofiber webs with significant antifungal activities. However, we were not able to prepare nanofibers with the OLE concentrations above $15 \%(\mathrm{w} / \mathrm{v})$ with desired nanofiber morphology. In brief, results given in Figure 5 showed that OLE used in this study had dominated effect on bacteria than fungi at the same concentration value.

To better understand the release mechanism of OLE from the nanofiber structure, it is necessary to estimate the strength of the bonds between extract and polymer molecules and where the extract is located inside the structure. In this study, the extraction using bead beater gave some clues about where OLE was located in nanofiber structure and how it was released. As seen in Figure 6, the increase in the amount of OLE in PBS solution was high in the first oscillation minutes during extraction with bead beater. Approximately $55 \%$ of OLE loaded to OLE-15 nanofibers and $\sim 51 \%$ of OLE loaded to OLE-12 nanofibers were released to the extraction medium at first $3 \mathrm{~min}$. This means the most of OLE was weakly integrated with polymers. It is likely that OLE that was weakly integrated to hydrophilic polymer and polymer groups was released to the medium at the beginning of extraction process, because in aqueous medium, active agents show a tendency to leave the structure easily depending on their ionic strength (Kim et al., 2004). As the oscillation progressed, OLE that was strongly integrated to the nanofiber structure and embedded in the structure was released to the PBS solution.

The release rates of the phenolics from the nanofibers can be adjusted by polymer molecular weight and concentration, interactions of polymer and active agent, shell and core feeding rates, high degradability of the polymers, active agent concentration, and porosity (Chakraborty et al., 2009). Burst release is related to the core components that diffuse in aqueous medium. It is thought that the diffusion that is actualized from the shell polymer does not affect the first release. The release occurred throughout the wall of shell polymer slows down the release rate and strong 
polymer-active component interactions come into prominence at the second stage that sustained release is occurred (Srikar et al., 2008).

HA is an anionic polysaccharide that is hydrophilic. SF has both hydrophilic and hydrophobic groups. OLE is a water-soluble compound. The burst release observed in first $72 \mathrm{~h}$ is related to weak and limited interactions between the hydrophilic polymer or polymer groups and extract (Fig. 7). The following sustained release belongs to slow wetting behaviors of hydrophobic groups, as a result of surface characteristics as stated by $\mathrm{Hu}$ et al. (2010). The remaining parts of OLE that were not released from both of the nanofibers can be explained by the strong bonds and interactions occurred between the extract and polymers.

The total antioxidant activities of plant-extractloaded structures are important in terms of using them as tissue scaffolds providing therapeutic effects. The total antioxidant activity analysis performed according to TEAC method indicated that the bioactivity of OLE was retained during electrospinning and no negative effect was observed on OLE by blending it with HA. OLE-15 nanofiber web released higher amount of OLE loaded to its structure than that of OLE-12 nanofiber web and it showed higher antioxidant activity compared to OLE-12 nanofiber web, as seen in Figure 9.

When the results of HPLC analysis were evaluated (Fig. 11 and Table 1), it was observed that flavones such as luteolin-7-glucoside, apigenin-7-glucoside, diosmetin-7-glucoside, and vanillic acid have not been released to medium in significant quantities. On the other hand, the percentages of polar phenolic compounds such as hydroxytyrosol, tyrosol, and catechin that have been released from nanofibers were greater than those of OLE directly dissolved in PBS solution (percentages given in bold in Table 1). This can be explained by the conversion of nonpolar components of OLE into polar ones due to the contact of extract with shell solution solvent (formic acid) during spinning. It is known that treatment of phenolic compounds with strong acids convert nonpolar components to polar components. $\mathrm{H}$ bonding is responsible for bonding of polar phenolic compounds to polymer (Prigent, 2005). Breakage of $\mathrm{H}$ bondings in aqueous medium and limited physical interactions between hydrophilic compounds and polymer cause release of polar phenolic components into the medium (Maretschek et al., 2008). These polar components arise from nanofibers are more bioactive than oleuropein (Benavente-Garcia et al., 2000; Bisignano et al., 1999). Therefore, degradation of phenolic components to more polar ones provides advantages in terms of efficiency of antimicrobial and antioxidant activity.

While some of the phenolic components of OLE degraded into polar compounds, some of them may have chosen to strongly bond to fibroin molecules. This is because hydrophobic phenolic components prefer to bond to protein molecules by hydrophobic interactions in an aqueous medium (Tang et al., 2003; Baycin et al., 2007). Flavones that have retention times close to oleuropein may be interacted with SF selectively. This might implies that a selective release mechanism took place in the release of phenolic compounds of OLE from SF/HA nanofiber webs.
By the help of FTIR analysis performed on nanofiber web samples before release test, it was observed that majority of the SF was in $\alpha$-helix structure (Fig. 12). This could be attributed to inadequate crystallization due to the fast solvent evaporation and short flight times of fibers (Wang et al., 2004). An extra crosslinking treatment may be performed to increase the amount of $\beta$-sheet structures. When the bands in the spectrum of nanofibers after release test are examined (Fig. 14), an increase in the density of the wide band at wavelength of $3300 \mathrm{~cm}^{-1}$ (NH stretching) is seen. Before release test, characteristic bands of $\alpha$-helix conformation (Amid I and Amid II) were observed at wavelengths of 1651 and $1637 \mathrm{~cm}^{-1}$, respectively. After release test, characteristic bands of $\beta$-sheet conformation (Amid I and Amid II) were observed at wavelengths of 1635 and $1521 \mathrm{~cm}^{-1}$, respectively. This clearly shows that water-soluble $\alpha$-helix structures were removed from the nanofibers and $\beta$-sheet structures remained.

Since it is obvious from the release analysis of total phenolic content from the nanofibers, OLE-12 nanofiber webs still contain $\sim 30 \%$ of OLE loaded to their structure after a release period of 1 month. This is observed as a band at a wavelength of $1075 \mathrm{~cm}^{-1}$ related to $\mathrm{C}=\mathrm{O}$ stretching of phenols (Fig. 14). These phenolic compounds were probably hydrophobic phenolic contents strongly bonded to SF.

There are limited numbers of functional groups in polymers that components can bond to. If the bondable phenolic component molecule number is equal to that of functional groups, all components bond to those groups. However, if the phenolic component molecules are much more than bondable functional groups, components may be easily transferred to release medium. When phenolic component/polymer concentration ratio decreases, the probability of formation of multimeric structures between phenolics and polymer molecules by cross-linking increases (Prigent, 2005; Siebert et al., 1996). So, the high release ratio observed in OLE-15 nanofibers is related to release of monomeric structured phenolics as small bulks. Nonreleased phenolics of OLE-12 nanofibers are related to low ratio of phenolic component/polymer concentration, formation of multimeric structures promoted by cross-linking.

Weight-loss analysis confirmed the degradation of some hydrophilic parts of SF during in vitro batch release test. It is known that $\alpha$-helix structures degrade faster than $\beta$-sheet structures (Lu et al., 2011; $\mathrm{Hu}$ et al., 2011). SEM images of all nanofibers (Fig. 15) point out that there was a swelling mechanism during release test. The minimum swelling values observed in OLE-12 nanofibers may be explained by the encapsulated extract that did not replace with PBS and decrease in hydrodynamic diameter of fibroin due to the bonds between SF and phenolic compounds of OLE. As seen from SEM images, some of the hydrophilic groups of SF were dissolved and OLE was released not only from the core regions of nanofibers but also through the pores of shell walls. It is believed that there are more pores, but they cannot be easily seen because of fusing (created by degradation of some polymer parts). The minimum fusing was observed in OLE-15 nanofibers due to the maximum weight loss of aforementioned nanofiber (Fig. 15c). 


\section{CONCLUSIONS}

Fabrication of biomaterials by electrospinning has gained great attention in the last years. This method provides an opportunity to produce completely natural products. In this study, coaxial nanofibers with an SF (sheath)/HA+OLE (core) structure were successfully fabricated. A natural bioactive (antimicrobial and antioxidative) compound, OLE, was utilized to prepare coaxial nanofiber webs. Significant antibacterial, but rather poor antifungal activities were observed against test microorganisms. The results also showed that as the OLE content of nanofibers increase, their growth inhibition ability of increases as well. The results of total antioxidant activity and total phenolic content analyses revealed that the sheath/core structured nanofibers prepared in this study had antioxidant activities and these activities were retained during preparation process. Moreover, polar phenolic contents of OLE that exhibit higher antioxidant activity were selectively released from nanofiber webs. Overall results indicated that this novel biomaterial is a promising candidate for biomedical applications. Further studies will be conducted to examine the cytocompatibility of these nanofibers in order to expose their potential to be used as novel products for scaffolding and drug release applications.

\section{ACKNOWLEDGMENTS}

The authors gratefully acknowledge the DUAG Natural Products for their support.

\section{REFERENCES}

Altıok E, Baycın D, Bayraktar O, Ulku S. 2008. Isolation of polyphenols from the extracts of olive leaves (Olea europaea L.) by adsorption on silk fibroin. Sep Purif Tech 62:342-348.

Altman GH, Diaz D, Jakuba C, Calabro T, Horan RL, Chen J, Lu H, Richmond J, Kaplan DL. 2003. Silk based biomaterials. Biomaterials 24:401-416.

Baycın D, Altıok E, Ulku S, Bayraktar O. 2007. Adsorption of olive leaf (Olea europaea L.) Antioxidants on silk fibroin. J Agric Food Chem 55:1227-1236.

Benavente-Garcia O, Castillo J, Lorente J, Ortuno A, Del Rio JA 2000. Antioxidant activity of phenolics extracted from Olea europaea L. Leaves. Food Chem 68:457-462.

Bisignano G, Tomaino A, Cascio RL, Crisafi G, Uccella N, Saija A. 1999. On the in vitro antimicrobial activity of oleuropein and hyrdoxytyrosol. J Pharm Pharmacol 51:971-974.

Briante R, Patumi M, Terenziani S, Bismuto F, Febbraio F, Nucci R. 2002. Olea europaea L. Leaf extract and derivatives: antioxidant properties. J Agric Food Chem 50:4934-4940.

Chakraborty S, Liao I-C, Adler A, Leong KW. 2009. Electrohydrodynamics: A facile technique to fabricate drug delivery systems. Adv Drug Delivery Rev 61:1043-1054.

Chen C, Chuanbao C, Xilan M, Yin T, Hesun Z. 2006. Preparation of non-woven mats from all-aqueous silk fibroin solution with electrospinning method. Polymer 47:6322-6327.

Chew SY, Wen J, Yim EKF, Leong KW. 2005. Sustained release of proteins from electrospun biodegradable fibers. Biomacromolecules 6 : 2017-2024.

Fischer RL, McCoy MG, Grant SA. 2012. Electrospinning collagen and hyaluronic acid nanofiber meshes. J Mater Sci Mater Med 23: 1645-1654.

Hang Y, Zhang Y, Jin Y, Shao H, Hu X. 2012. Preparation of regenerated silk fibroin/silk sericin fibers by coaxial electrospinning. Int $J$ Biol Macromol 51:980-986.

Haxaire K, Marechal Y, Milas M, Rinaudo M. 2003. Hydration of polysaccharide hyaluronan observed by IR spectrometry. I. Preliminary experiments and band assignments. Biopolymers (Biospectroscopy) 72:10-20.

Hu W, Huang Z-M, Liu X-Y. 2010. Development of braided drugloaded nanofiber sutures. Nanotechnology 21:11p
Hu X, Shmelev K, Sun L, Gil E-S, Park S-H, Cebe P, Kaplan DL. Regulation of silk material structure by temperature-controlled water vapor annealing. Biomacromolecules 12:1686-1696.

Ji Y, Ghosh K, Shu XZ, Li B, Sokolova JC, Prestwich GD, Clark RAF, Rafailovicha MH. 2006. Electrospun three-dimensional hyaluronic acid nanofibrous scaffolds. Biomaterials 27:3782-3792.

Jiang H, Wang L, Zhu K. 2014. Coaxial electrospinning for encapsulation and controlled release of fragile water-soluble bioactive agents. J Cont Release 193:296-303.

Jin G, Prabhakaran MP, Kai D, Annamalai SK, Arunachalam KD, Ramakrishna S. 2013. Tissue engineered plant extracts as nanofibrous wound dressing. Biomaterials 34:724-734.

Jin H-J, Chen J, Karageorgiou V, Altman GH, Kaplan DL. 2004. Kaplan, Human bone marrow stromal cell responses on electrospun silk fibroin mats. Biomaterials 25:1039-1047.

Jin H-J, Fridrikh SV, Rutledge GC, Kaplan DL. 2002. Electrospinning Bombyx mori silk with poly(ethylene oxide). Biomacromolecules 3:1233-1239.

Kim K, Luu YK, Chang C, Fang D, Hsiao BS, Chua B, Hadjiargyrou M. 2004. Incorporation and controlled release of a hydrophilic antibiotic using poly(lactide-co-glycolide)-based electrospun nanofibrous scaffolds. J Cont Release 98:47-56.

Kim K-H, Jeong L, Park H-N, Shin S-Y, Park W-H, Lee S-C, Kim TI, Park Y-J, Seol Y-J, Lee Y-M, Ku Y, Rhyu I-C, Han S-B, Chung C-P. 2005. Biological efficacy of silk fibroin nanofiber membranes for guided bone regeneration. J Biotech 120:327-339.

Li C, Vepari C, Jin H-J, Kim HJ, Kaplan DL. 2006. Electrospun silkBMP-2 scaffolds for bone tissue engineering. Biomaterials 27: 3115-3124.

Li S, Wu H, Hu X-D, Tu C-Q, Pei F-X, Wang G-L, Lin W, Fan H-S 2012. Preparation of electrospun PLGA-silk fibroin nanofibersbased nerve conduits and evaluation in vivo. Artif Cells Blood Substitut Biotechnol 40:171-178.

Loh XJ, Peh P, Liao S, Sng C, Li J. 2010. Controlled drug release from biodegradable thermoresponsive physical hydrogel nanofibers. J Cont Release 143:175-182.

Lu Q, Zhang B, Li M, Zuo B, Kaplan DL, Huang Y, Zhu H. 2011. Degradation mechanism and control of silk fibroin. Biomacromolecules 12:1080-1086.

Luis A, Gil N, Amaral ME, Duarte AP. 2012. Antioxidant activities of extracts from Acacia melanoylon, Acacia dealbata and Olea europaea and alkaloids estimation. Int J Pharma Pharmaceut Sci $4: 9 \mathrm{p}$.

Maretschek S, Greiner A, Kissel T. 2008. Electrospun biodegradable nanofiber nonwovens for controlled release of proteins. J Cont Release 127:180-187.

Markin D, Duek L, Berdicevsky I. 2003. In vitro antimicrobial activity of olive leaves. Mycoses 46:132-136.

Min B-M, Lee G, Kim SH, Nam YS, Lee TS, Park WH. 2004. Electrospinning of silk fibroin nanofibers and its effect on the adhesion and spreading of normal human keratinocytes and fibroblasts in vitro. Biomaterials 25:1289-1297.

Moghe AK. 2008. Core-Sheath Differentially Biodegradable Nanofiber Structures for Tissue Engineering. PhD Thesis. The Graduate Faculty of North Carolina State University. 176 p.

Okuda T, Tominaga K, Kidoaki S. 2010. Time-programmed dual release formulation by multilayered drug-loaded nanofiber meshes. J Cont Release 143:258-264.

Prigent S. 2005. Interactions of phenolic compounds with globular proteins and their effects on food-related functional properties. $\mathrm{PhD}$ thesis. Wageningen University. $133 \mathrm{p}$.

Saraf A, Baggett LS, Raphael RM, Kasper FK, Mikos AG. 2010. Regulated non-viral gene delivery from coaxial electrospun fiber mesh scaffolds. J Cont Release 143:95-103.

Shao S, Li L, Yang G, Li J, Luo C, Gong T, Zhou S. 2011. Controlled green tea polyphenols release from electrospun PCL/MWCNTs composite nanofibers. Int J Pharma 421:310-320.

Siebert KJ, Troukhanova NV, Lynn PY. 1996. Nature of polyphenolprotein interactions. J Agric Food Chem 44:80-85.

So Y, Mo X. 2011. Dual drug release from coaxial electrospun nanofibers. J Cont Release 152:e82-e84

Srikar R, Yarin A-L, Megaridis CM, Bazilevsky AV, Kelley E. 2008. Desorption-limited mechanism of release from polymer nanofibers. Langmuir 24:965-974.

Sudjana AN, D’Orazio C, Ryan V, Rasool N, Ng J, Islam N, Riley TV, Hammer KA. 2009. Antimicrobial activity of commercial Olea europaea (olive) leaf extract. Int J Antimicrob Agents 33:461-463.

Tang HR, Covington AD, Hancock RA. 2003. Structure-activity relationships in the hydrophobic interactions of polyphenols with cellulose and collagen. Biopolymers 70:403-413. 
Um IC, Fang D, Hsiao BS, Okamoto A, Chu B. 2004. Electrospinning and electroblowing of hyaluronic acid. Biomacromolecules 5:14281436.

Visioli F, Galli C. 1998. Olive oil phenols and their potential effects on human health. J Agric Food Chem 46:4292-4296.

Wang D, Liu H, Fan Y. 2015. Silk fibroin for vascular regeneration. Microsc Res Tech.

Wang M, Jin HJ, Kaplan DL, Rutledge GC. 2004. Mechanical properties of electrospun silk fibers. Macromolecules 37: 6856-6864.
Wang Q, Xiong J, Zhang H, Li N, Xie J, Liu G. 2011. Preparation and properties of PBS-SF core-shell composite ultrafine fibrous membranes by coaxial electrospinning. J Acta Materiea Compositae Sinca 28:88-93.

Xu X, Chen X, Xu X, Lu T, Wang X, Yang L, Jing X. 2006. BCNUloaded PEG-PLLA ultrafine fibers and their in vitro antitumor activity against Glioma C6 cells. J Cont Release 114:307-316.

Young DS. 2006. Hyaluronic Acid-based Nanofibers via Electrospinning. MSc Thesis. Graduate Faculty of North Carolina State University. $97 \mathrm{p}$. 